\title{
23. Social safety nets are crucial to the COVID-19 response: Some lessons to boost their effectiveness
}

\section{Daniel Gilligan}

The twin health and economic shocks of the COVID-19 pandemic are staggering in their breadth and scale. While the disease arrived later and has spread more slowly in many low- and middle-income countries, COVID-19 is threatening the lives and long-term livelihoods of millions of poor people, and could push an additional 140 million into extreme poverty.

We know from past crises that while an economic shock's impacts may vary across the income distribution, the poor face a multitude of vulnerabilities to the pandemic's effects. In many places, the poor are more likely to have underlying or untreated health conditions (such as hypertension, diabetes, and vitamin D deficiency) which raises the risk of serious illness if they are exposed. Poor households are also more likely to cope with income loss by selling productive assets or undertaking work that is either inherently riskier (for example, construction or sex work) or increases their risk of coronavirus exposure. Their children may also be less likely to return to schools upon reopening, permanently reducing their earning potential. These factors leave the poor even more vulnerable to additional impending shocks, like desert locusts in the Horn of Africa or cyclones in South Asia.

Targeted social safety nets for the poor are central to the effort to stifle these negative impacts and protect the substantial gains made globally in the fight against poverty, food insecurity, and malnutrition in this century. Why are social safety nets important to the pandemic response, what challenges do they face, and what lessons can we glean from past research into social protection programs to craft effective responses over the long term?

Social safety nets have played a major role in the response to the COVID-19 pandemic in the last three months. According to an effort by Ugo Gentilini of the World Bank to track social protection responses during the crisis, 190 countries have implemented, adapted, or planned over 900 social protection measures during the crisis, often in the form of cash transfers. This response has included an expansion in the number of social protection beneficiaries by roughly $15 \%$ in South Asia and East Asia and the Pacific, but by only $2 \%$ in the Africa region. Several countries have offered a temporary sharp increase in the benefit amount for current beneficiaries.

\section{Why social safety nets during a health crisis?}

Three factors explain why social safety nets have been central to the COVID-19 response. First, two decades of extensive, rigorous research on social protection programs has documented their effectiveness at protecting food security, assets, and human capital, including in a crisis. Safety nets can 
also improve health, including for newborns, through improved nutrition when combined with complementary nutrition programs, a high policy priority during the pandemic.

Second, large-scale transfers help to replace lost income for credit-constrained poor households and counter the economic drag of the pandemic by providing a fiscal stimulus that, under certain conditions, may generate positive multiplier effects during the recovery. A temporary increase in cash transfers during the pandemic can also make moral and political sense, protecting those most in need and building trust in government.

Third, the infrastructure of a social safety net was already in place, to varying degrees, in most countries. Over the last two decades, social protection, and particularly social assistance, has grown in popularity as a leading response to poverty. Many poor countries developed targeted in-kind and cash transfer programs, expanding their roles and improving their efficiency. Many safety net programs are designed to be "shock responsive": During a 2011 drought, Ethiopia increased benefits for $85 \%$ of the 7.6 million beneficiaries in the Productive Safety Net Program (PSNP) and provided temporary benefits to an additional 3.1 million people. As the COVID-19 pandemic hit earlier this year, many countries had the payment mechanisms, poverty registries, and local selection committees in place to quickly expand benefits and renew program targeting.

\section{Challenges ahead}

For countries that have had initial successes using social safety nets to respond to the pandemic, significant challenges remain. For most, the fiscal cost is the greatest of these; continuing transfers beyond a few months will be difficult. Yet the pandemic and its economic consequences may drag on, or return later this year after a failed reopening. Social distancing measures pose obstacles to targeting new beneficiaries, as this typically involves in-person interviews or local committees meeting to screen applicants. Delivering cash or in-kind transfers can also increase the risk of coronavirus transmission, and most programs still deliver benefits in ways requiring person-to-person contact.

\section{How should social safety nets be designed for the pandemic?}

Evidence from past research on the design, implementation, and effectiveness of social assistance programs during an economic crisis provides guidance on handling some of these challenges:

1. When safety net programs are disrupted, maintain transfers by adopting alternative delivery methods. At the start of the pandemic, many school feeding programs ceased operating as countries closed schools. These are one of the most popular forms of social assistance, reaching more than 270 million children in 89 countries in one World Bank estimate. In India, many school systems replaced on-site meals with rations delivered to homes or available for pick-up at schools. As they reopen, schools should consider maintaining take-home rations initially, to reduce the risk of coronavirus transmission when students eat together; in Uganda, fortified take-home rations were as effective as equivalent on-site school meals for improving school participation and attainment, and for reducing anemia prevalence for adolescent girls. 
2. Eliminate conditions on assistance temporarily. Reassess their importance and effectiveness during the recovery. Cash transfers are often conditioned on child school participation or adult work requirements. These should be suspended during the pandemic. Unconditional cash transfers can also improve school attendance. In Ethiopia, PSNP work requirements were temporarily suspended for beneficiaries because it was no longer possible to bring work teams together safely. Payments to public works beneficiaries continued, and were even accelerated in case it became impossible to make payments for some time. When work can resume, programs should identify work activities that bolster the COVID-19 response, such as building or renovating health clinics or supplying hand-washing stations.

3. Strengthen and expand targeting. Safety nets often exclude a high proportion of the poorest households and fail to reach the most vulnerable groups. They should expand coverage in poor areas and make efforts to target migrants, orphans, and the urban unemployed, who face substantial livelihood risks and have limited social support. Social assistance programs should also be used as platforms to identify the newly poor. For example, community health systems and local government can be used to target new beneficiaries. In areas that are particularly hard-hit with a collapse in employment, programs should consider providing universal transfers temporarily.

4. The form of assistance matters, but distributing it quickly is the priority. Food, vouchers, or cash assistance can all increase household consumption and improve calorie intakes, though vouchers may perform better than food transfers or cash at improving measures of dietary diversity and thus quality. However, setting up a voucher system involves coordination with food retailers and thus may be challenging to establish during the pandemic. Cash transfers have many of the advantages of voucher programs - they work well where people have access to markets and as long as prices do not escalate - and they are usually cheaper to deliver. Still, all of these transfer modalities can be beneficial, so following a "no regrets policy" is best: Prioritize speed in scaling up transfers with whatever method works the fastest.

5. Respond to the crisis with the future in mind. Many acknowledge the need to "build back better" following the pandemic to improve safety net systems. Here are some priorities:

- Strengthen mental health services for beneficiaries. Anxiety and depression increase with poverty and, in women, are associated with worse development outcomes for their children. The pandemic is an opportunity to bring mental health out of the shadows. Programs should build a cadre of community health workers to provide mental health services and screening.

- Design new programs or features to be gender-sensitive. Most social assistance programs are not designed with the specific needs of women beneficiaries in mind, a wasted opportunity that can widen gender inequality. As my colleagues Melissa Hidrobo, Neha Kumar, Tia Palermo, Amber Peterman, and Shalini Roy emphasize, social protection programs designed to be gender-sensitive during the pandemic have the potential to protect women's livelihoods and assets, reduce unequal burdens of care, improve women's empowerment, and reduce intimate partner violence.

- Strengthen nutrition-sensitive social assistance. An ever-growing body of evidence shows that critical investments in health and nutrition for the poor can be better achieved by combining 
cash transfers with information campaigns and access to services. These investments in child human capital will have large economic returns as these individuals enter the labor market and begin their own families.

- Invest more in mobile payments. Contactless mobile payments have a clear advantage during the pandemic. Though the poor continue to have less access to mobile phones, it is important to provide transfers through mobile phones when possible - or better yet, to steeply subsidize mobile phone ownership for the poor. Many will benefit from the new access to social networks and information. Even where literacy is low, household heads often have a family member who can support the phone use.

6. Strengthen fiscal support for the social assistance response. The global slowdown in economic activity and lockdowns have ravaged economies of low- and middle-income countries at a time when many face a debt crisis. With public health costs growing enormously, governments will need aid to finance a robust social safety net response.

As they try to contain the pandemic, countries must also confront a rise in extreme poverty and the suffering that goes along with it. But they have many tools to combat this problem. Extensive research on social assistance programs provides a firm foundation for approaches to strengthen safety net designs and adapt responses to fit changing circumstances. These actions can improve social safety nets and help to counter some of the worst effects of the COVID-19 pandemic.

Originally published June 18, 2020. 